Contents List available at VOLKSON PRESS
Education, Culture and Social Development (ECSD)
DoI : http://doi.org/10.26480/icecsd.01.2018.56.59
Journal Homepage: $:$ https://topicsonsocialdevelop.com/

\title{
A COGNITIVE POETICS INTERPRETATION OF ZEN IN WANG WEI'S LANDSCAPE POEMS
}

\author{
Zhong Deng*, Hongmei Zhang \\ School of Foreign Languages, Southwest Minzu University Chengdu, China \\ *Corresponding Author E-mail: icomehere@126.com
}

This is an open access article distributed under the Creative Commons Attribution License, which permits unrestricted use, distribution, and reproduction in any medium, provided the original work is properly cited

\section{ARTICLE DETAILS}

\section{Article History:}

Received 12 November 2017 Accepted 12 December 2017 Available online 1 January 2018

\section{ABSTRACT}

\begin{abstract}
Cognitive poetics provides a refreshed alternative of understanding literature by applying the burgeoning cognitive science, in particular, cognitive linguistics and cognitive psychology to the interpretation of literary texts. In this paper, we mainly apply the figure-ground theory, one of the most important approaches in cognitive poetics, to the interpretation of Zen in Wang Wei's landscape poems. Based on the poetic language which profiles spatial distribution and arrangement, we find Zen is derived from the uncertainty and ambiguity of specific spatial distribution and configuration characteristics. We believe this interpretation serves a desirable means to promote the current scholastic understanding of Zen in Wang Wei's poems.
\end{abstract}

\section{KEYWORDS}

Cognitive poetics, landscape poems, Zen, figure-ground theory.

\section{INTRODUCTION}

Much research has been done on Wang Wei's landscape poetry and Zen, in particular, in the poems. A researcher analyzed Zen and its aesthetic value through detailed explanations of Wang Wei's family and educational background [1]. Another researcher presented the imagery of Zen on the basis of traditional Chinese imagery theories [2]. Based on a study, Zen more deeply by integrating Wang Wei's educational background with his proficiency in Buddhism and Taoism [3]. A researcher provided detailed accounts of Zen by presenting a critical review from a western perspective [4]. However, such studies are not satisfying since the interpretations of Zen are ambiguous and vague as a result of their preoccupation with a well-established Zen in Buddhism. In other words, Zen has been exclusively interpreted as a fixed concept extended from Buddhism into literature. Thus, their studies may fail to specify denotations and connotations of Zen peculiar to landscape poems, while teeming with obscure and awkward Buddhist terms which not only perplex readers but also deviate from the the literariness of the original poetry. Therefore, this paper attempts to provide a new interpretation of Zen in the context of Wang Wei's landscape poetry. More specifically, we primarily apply the figure-ground theory to interpret Zen as a representation of the ambiguity and uncertainty of specific spacial distribution and configuration characteristics.

\section{THEORETICAL FRAMEWORK}

\subsection{A Brief Introduction to Cognitive Poetics and Figure-ground Theory}

According to a research, cognitive poetics is concisely defined as systematic applications of cognitive science to literary studies [5]. It offers a reconnection of literary scholarship with natural readers [6]. Guided by such a rationale, scholars of cognitive poetics have developed varieties of perspectives and approaches. He contended that applying relevant theories of cognitive linguistics to the literature analysis enables readers to detect why they made a particular understanding while reading. A scholar furthered this view by claiming that the potential of cognitive poetics lies in discovering what was neglected, that is, proposing new reasons for unknown effects, implications and aesthetic feelings [7]. As a cognitive view, the figure/ground theory claims that people are so heavily focused on significant features and patterns of objects that they tend to ignore insignificant ones. Besides, the gestalt psychologists concentrating on perception research found that perceptual fields can be divided into the figure and the ground [8]. Namely, the foreground that easily attracts people's attention and stays more prominent among other objects is called the figure, while the ground, also called background serves as a foil to the figure. Precisely, the figure is systematically shaped, well-structured, very coherent and more prominent, but the ground lacks shapes, structures and coherence [9]. As a result, the figure is apt to be noticed and recognized, while the ground is vague and undifferentiated, serving as a reference to the figure.

\subsection{An Introduction to Zen and its Relationship with Landscape} Poetry

Wang Wei lived in the most prosperous dynasty in China's monarchy history when Buddhism with Zen at its core and traditional Chinese culture were mingling with each other. In other words, Buddhism thoroughly penetrated into every aspect of Chinese culture and profoundly impacted Chinese literature, particularly the philosophy of poetry and literary creation [10]. With subjective idealism at its core philosophy, Chan (Zen) sect holds that all the objective things are illusions and phantoms reflected by hearts and derived from subjective spirits. Furthermore, the way of practicing Zen is meditation through which the truths and religious beliefs can be instantly comprehended and procured in the form of being the identity of both outside things and inside hearts. Besides, the insight theory plays an essential role in Chan sect philosophy. The insight theory involves instant perception of unreal things and complete recognition of one's nature and conscience [11]. Through insight learning, people are likely to extricate from the worldly affairs and traditional doctrines. As to the relations between Zen and landscape poems, two classics the Book of Songs and The Songs of Chu demonstrated that mountains, rivers, flowers and other natural images employed in poetry are the bonds and bridges for the poets expressing emotions and feelings. Taoists hold that nature and people can be reciprocally transformed. Nature can be personified just as people can be equally converted into nature. On the contrary, Buddhists contend that the essence of Zen can be acquired through meditation and 
proximity to nature [12]. Obviously, Buddhists grasp the decree of Zen through observations and meditation on natural scenery in contrast with the Taoists' identity of people and natural scenery. Hence, there is a strong interdependence between Chan sect and landscape poetry. In terms of the relationship between Buddhist nature and natural images, many an eminent monk have lived away from worldly affairs to practice Zen and gain insight through meditation because they believed that Buddhist nature is embodied in natural images [13]. The Chan sect considers that Buddhist nature is nameless and shapeless beyond any descriptions. Some natural images bearing characteristic transparency, emptiness and purity can be the carrier of the Buddhist nature. In the landscape poetry, the moon conveying well-rounded Buddhist nature, pine trees representing the embodiment of spiritual Buddha, white clouds revealing the purity and cleanness and blue sky demonstrating the eternality and instancy often constitute a closed or open space. These images act as a bond and a bridge to convey Buddhist nature.

\section{CASE STUDY: SHANJUQIUMING}

Based upon our language-level analysis as a starting point for a cognitive study guided by the figure/ground theory, we find that Wang Wei's landscape poetry has two main characteristics in spacial distribution and configuration. Firstly, the image of the poetry is a symbolically vast and empty space as the center of landscape which is eternal and beyond the time limit. Shanjuqiuming is one of the examples. Secondly, landscape of the poetry generally consists of white cloud, blue sky and azure dome, characterized by all-inclusive sense of space. Zhongnanshan falls into this category. Now, we will analyze the lines that profiles the spacial distribution in an attempt to interpret the Zen with the figure/ground theory with the two poems as examples.

\section{Autumn Evening in the Mountains}

After fresh rain in mountains bare Autumn permeates evening air.

Among pine-trees bright moonbeams peer; Over crystal stones flows water clear.

Bamboos whisper of washer-maids; Lotus stirs when fishing boat wades

Though fragrant spring may pass away, still here's the place for you to stay.

\subsection{Uncertainties}

\subsubsection{Uncertainties of the Spacial Distribution and Configurations of Images}

Considering the poem as a whole, there are twelve images in the three lines, the mountain, the rain, the evening air and the autumn, the moonbeams, the pine trees, the water and the stones, the whispers, the washer-maids, the lotus and the fishing boats. The last line depicts the poet's feelings and emotions about the scene. According to the English version by $\mathrm{Xu}$ Yuanchong, we can figure out one possible spacial distribution of these images. The fresh rain over the mountain, the autumn permeating the evening air, the moonbeams floating among the pines trees, the water flowing over the crystal stones, the washer-maids whispering and the lotus stirring the fishing boats, all of this compose an array of natural landscapes. However, we cannot determine what the poet intends to highlight or overshadow. That is, interpretations to spacial arrangements of these images vary among people with diverse cognitive styles or experiences. Hence, uncertainties of spacial distribution and configurations are all down to myriads of interpretations.

\subsubsection{Uncertainties of Implications of the Modifiers}

In the first line, there are two noticeable modifiers. Kong has ten definitions based on two different tones. Xin has seven definitions in the New Century Chinese-English Dictionary. It can be defined as follows. When Kong pronounced as level tone;

1) Empty; hollow; void; vacant; unoccupied; (of the content of a book) lacking substance; impractical.

2) For nothing; in vain; vainly.

3) there is not; be without.

When Kong pronounced as falling tone;
1)Leave empty and blank; empty.

2)Unoccupied; unfilled; vacant.

\section{3) Empty space.}

Xin can be defined as following meanings with level tone.

1)Appearing for the first time; new; fresh.

2)Make new.

3)Unused; new.

4) Recently married or just being married.

In this poem, several definitions of Kong and Xin can be mobilized to describe the images. Various definitions of these modifiers lead to unfixed interpretations thus causing uncertainties in spacial distribution.

\subsubsection{Uncertainties of Images}

In the whole poetry, there are some perspicuous natural images, such as, the mountain and the rain, of which modifiers are indefinite in traditional Chinese landscape paintings can be interpret as various forms directly or indirectly. The poets intends to vacate further space for readers to conceive different shapes, sizes, colors and other physical and insubstantial properties of these natural images. And various definitions of the modifiers thus lead to diverse images. Therefore, the images bear their uncertainties. We can regard the poem itself more an inexhaustible and boundless ore source than a matter of opinions. It's like a primitive forest with such a strong regeneration capacity and sustainability that so far no one can figure out how much areas it covers, how many species of trees it bears. Our exploitation to it is endless and unlimited. In the bountifulness and profusion lies the mystery of the forest as the excellence of the poetry lies in the uncertainties and indefiniteness.

\subsection{Highlighting the Words or Phrases related to Zen}

\subsubsection{Words that Express Emptiness}

Kong is the modifier of the image, the mountain. With a train of properties, such as shape, color, size and height, the poet applies only one word to depict emptiness of the mountain. According to the figure/ground theory, all the characteristics of the mountain are well-structured. However the poet sets the shape, color, size, height and other properties of the mountain as the ground among which the emptiness and tranquility as the figure are emphasized. Kong pronounced both in level tone and falling tone has the meaning of emptiness and vacancy, precisely conveying quintessence of Zen. In Chinese traditional landscape paintings, the painter tends to spare much vacant space on his painting as the poet vacate further unoccupied space for readers. Then how does the Kong connect with Zen? We have mentioned in the introduction part the poet was immersed himself in Buddhism in his early ages. Taoism maintains that the origin of the universe is nothingness and voids with steaming mists as the Tao Te Ching said, 'Yet Heaven and Earth and all that lies between is like a bellows. In that it is empty but gives a supply that never fails. The Buddhism claims that a void is Buddhist nature and all objects in the universe are as fragile as bubbles. It is shapeless and endless emptiness that embodies pure and clear nature of the world. One only making himself clean and unoccupied is the making of a Buddhist with the capability of comprehending Zen. In the poetry, the empty and peaceful mountain mirrors poet's inner thoughts and minds.

\subsubsection{Words that express cleanness and purity}

In the poetry, the moon, the pine trees and the lotus are evident natural images. There are myriads of natural images above or below, dynamic or static. However, the poet sorts out meticulously the moon, the pine trees and the lotus. Unreachable distances and obscured moonbeams endow the moon with serenity and tranquility. Furthermore in Buddhism, the full moon symbolizes well-rounded Buddhist nature. The tenacity of pine trees are found in the adverse growing environment. The lotus that rises out of the silt demonstrates its cleanness and purity as the pure Buddhist rises out of worldly affairs. These Buddhist-related images occasionally make a conquest among poets and painters in past and present, Wang Wei without exception. Based on the figure/ground theory, all natural images in the universe are set as a foil in this poetry, while the moon, the pine trees and 
the lotus emerging as the figure are highlighted and emphasized by the poet. The qualities the images conceive coinciding with thoughts of Zen are reflections of the poet himself. To practice the principles of Buddhism and cultivate the morality require a pure heart and few desires unequivocally consistent with the characteristics of the three images.

\subsection{Highlighting Daily Activities of People in the Poem}

In the last two lines, the poet behaves like a photographer who shifts its focus from natural images to average people in the countryside and their daily activities. Whispers of the washer-maids and movements of the fishing boats, transferring from sounds to visions are highlighted as the figure, and the rest of the natural images in the poetry are overshadowed as the ground to form a sharp contrast between nature and people. In the last line, an image of the poet himself slowly appears under the focus of readers. Before that, the poet remains out of sight purposely for intensifying the identity of images. It seems that natural scenery moves themselves under the dominance of outside powers thus profoundly impressed readers. However, the scenery is arranged under the possession of the poet's inner feelings and emotions. Only through the surface scenery can we detect the poet's deep emotional underflow. Consequently, the washer-maids and fishing boats are reversed as the ground, and the poet acting as an observer and a recorder of the scene is under the spotlight of readers as the figure. It is the poet himself who experiences the Zen-atmosphere scene. Though natural images carry and convey thoughts of Zen, it is the poet who interpret and experience Zen in his own. Clearly, the poet as the identity of natural images and people becomes an incarnation and exaltation of Zen.

\section{CASE STUDY: ZHONGNANSHAN}

Mountain Eternal South

The highest peak scrapes the sky blue; It extends from hills to the sea.

When I look back, clouds shut the view; When I come near, no mist I see.

Peaks vary in north and south side; Vales differ in sunshine or shade.

Seeking a lodge where to abide; I ask a woodman when I wade.

\subsection{Uncertainties}

\subsubsection{Uncertainties of Spacial Distribution and Configurations of Images}

The poetry depicts a landscape of Zhongnanshan Mountain microscopically and macroscopically. According to the English version by Xu Yuanchong, we can draw some clues about the spacial distribution. The Taiyi Mountain scrapes the sky and extends from hills to the sea; There are clouds, mists, peaks between two sides, vales and lodges. We can apply the figure/ground theory to the images that all these images are wellstructured and prone to get focused by readers but similarly all of them are shapeless and likely to be neglected by readers. One can treat the mountain, the clouds, the mists, the vales and the lodges as the figure just like one can regard all of them as the background decided by their own cognitive styles. It predicates that spacial distribution is unfixed in the poetry and there exists an ambiguity of the spacial arrangements among these images thus causing uncertainties.

\subsubsection{Uncertainties of Implications of the Modifiers}

In the first line, there are two remarkable modifiers. Jin has five following definitions based on two different parts of speech. When used as an adjective;

\section{1) Close; near; nearby; neighboring;}

2) Intimate;

3) Closely related;

4) Approximate; close to;

5) Easy to understand;

When it is used as a verb, it means "to approach";

Dao has four following definitions used as a verb:
1) Arrive; reach; get to;

2) Go to; leave for;

There are total five definitions of Jin and four definitions of Dao. And almost each of them is available to interpret different positional relationships between the peak and the capital, relationships between the mountains and the sea. And the range of the distance is indefinite and vague, so they bring out uncertainties and ambiguities in spacial distribution and arrangements.

\subsubsection{Uncertainties of Major Images}

In the second and third lines, there are clouds, mists, mountains, peaks and vales. The color of the clouds and mists is mentioned but unspecific. As we all know, there are a spectrum of colors in paintings in terms of tinge, texture and intensity. White and cyan are both general. Besides, the shape, location, brightness and other properties of the clouds and mists are ignored. The uncertainty of the properties equips everyone for regenerating the clouds and mists of all sizes and colors with their personal characteristics. It's the same with the mountains, the peaks and the vales. Readers can depict various peaks and various vales of different shapes, locations and colors. As a result, the poet intentionally more or less leaves the readers quite a lot space to recreate. Actually, the uncertainties and diverse interpretations reveal the excellence and eminence of the poetry which demonstrates its vigor and vitality.

\subsection{Emphasizing Words or Phrases Related to Zen}

In the second line, there are white clouds and blue mists which compose a clean and serene landscape. While there are loads of natural images, the poet preferentially screens out the white clouds and the blue mists. As the figure/ground theory holds, these two images with bright colors are coherent, more prominent and easy to get focused by readers. These two images are set as the figure to contrast with the endless and lush mountains acting as a foil to unmask vastness and wilderness of the mountain. According to the statistics, Wang Wei applied rather frequently the color of white for total ninety times to compose poetic imagery. Whiteness is simple but complex, for it contains spacious inclusiveness synchronously entails emptiness and remoteness. For one thing, it arouses brightness, tranquility, purity and elegance among impressions and emotions, simultaneously reminds people that all things are known eternally and are poignant in their transiency. For another, white clouds are a symbol of freedom in Buddhism. The fold or unfold clouds are mysterious experiences and symbolize the ease and free life style. Pure fleecy white clouds and swirls of mists constitute an inexplicable poetic imagery. Likewise, the white clouds and blue mists are two ordinary components of poet's life in seclusion.

\subsection{Highlighting People and their Daily Activities in the Poem}

In the last line, the poet acts as a photographer who alters its view from the distant to himself. The landscape of Zhongnanshan working as the ground, the image of the poet himself emerges on the stage and attracts the attention of readers as the figure. By doing so, vastness and remoteness of the mountain, purity and tranquility of the clouds and mists are converged on a sole image, the poet himself. Finally, real emotions and feelings of poet, hidden behind natural images exposes and we define that kind of inner emotional undercurrents as thoughts of Zen. In other words, it is the poet who experiences and immerses himself in the serene and quiet landscape, representing the way of comprehending Zen. The poet is an embodiment and even a sublimation of Zen.

\section{CONCLUSION}

To sum up, this paper applies the figure-ground theory in cognitive poetics to interpret Zen in Wang Wei's landscape poetry through spacial distribution and configuration of images, which differentiates the previous research on the same subject with the interpretation of poetry by preoccupation with established Zen. Thus this paper makes a definable achievement and has comparatively significant value for the cognitive poetics studies of ancient Chinese poetry. Specifically, we apply a new approach to interpreting Zen in Wang Wei's landscape poetry which, we found, correlates with the spacial distribution and configuration characteristics of images well situated in the cognition of people-nature relations peculiar to Chinese readers. Secondly, we believe this interpretation serves as a desirable means to promote the current 
scholastic understanding of Zen in Wang Wei's poems. Consequently, this research paradigm is worth popularizing since it is a relatively effective method of analyzing poetry with cognitive science, by means of which more readerly aspects of this poetry never found before with the traditional text analysis have been figured out. Unequivocally, cognitive poetics assists us in knowing not only the what happens in the literary works but why and how what happens can be perceived, understood and appreciated on the part of literature readers.

\section{REFERENCES}

[1] Yang, W.S. 2003. A Collection of Wang Wei's Poetry. Sichuan People's Press. 303.

[2] Xu, Y.C. 2001. 300 Tang Poems. China Higher Education Press.

[3] Du, R.Q. 2016. New Century Chinese-English Dictionary. Beijing: Foreign Language Teaching and Research Press.

[4] Du, R.Q. 2017. New Century Chinese-English Dictionary. Foreign Language Teaching and Research Press. 851.

[5] Du, R.Q. 2016. New Century Chinese-English Dictionary. Foreign Language Teaching and Research Press. 347.
[6] Gavins, J., Steen, G. 2003. Cognitive Poetics in Practice. London, Routledge.

[7] Lan, C. 2011. A Cognitive Approach to Tang and Song Poems. Foreign Language and Literature. 27 (1), 39-43.

[8] Li, H. 2009. An Intensive Study on Aesthetics of Tang Poems. Shanghai, Fudan University Press.

[9]Stockwell. 2002. Cognitive Poetics: An Introduction. London, Routledge.

[10] Xiong, M.Q. 2012. An Approach of Cognitive Poetics from Interpretations to Discoveries-Taking the Eagle for an Example, Foreign

[11] Language Teaching and Research, 44 (3), 448-457.

Yang, W.S. 2002. A Collection of Wang Wei's Poetry. Chengdu, Sichuan People's Publishing House.

[12] Yuan, X.P. 1980. On Zen and Picturesque in Wang Wei's Poetry. Social Science Front, (2), 276-283.

[13] Zhang, B.Z. 2008. Zen and Poetics. Beijing: People's Literature Publishing House. 